Institute for Computational Mathematics

Hong Kong Baptist University

ICM Research Report

09-14 


\title{
Superconvergence and Extrapolation Analysis of a Nonconforming Mixed Finite Element Approximation for Time-Harmonic Maxwell's Equations
}

\author{
Zhonghua Qiao * Changhui Yao $^{\dagger}$ Shanghui Jia ${ }^{\ddagger}$
}

\begin{abstract}
In this paper, a nonconforming mixed finite element approximating to the three-dimensional time-harmonic Maxwell's equations is presented. On a uniform rectangular prism mesh, superclose property is achieved for electric field $E$ and magnetic field $H$ with the boundary condition $E \times \mathbf{n}=0$ by means of the asymptotic expansion. Applying postprocessing operators, a superconvergence result is stated for the discretization error of the preprocessed discrete solution to the solution itself. To our best knowledge, this is the first global superconvergence analysis of nonconforming mixed finite elements for the Maxwell's equations. Furthermore, the approximation accuracy will be improved by extrapolation method.
\end{abstract}

Keywords: nonconforming mixed finite element; superconvergence; extrapolation; time-harmonic Maxwell's equations

AMS subject classifications: $65 \mathrm{M} 50,65 \mathrm{M} 60$

*Institute for Computational Mathematics \& Department of Mathematics, Hong Kong Baptist University, Kowloon Tong, Hong Kong. E-mail: zqiao@hkbu.edu.hk.

${ }^{\dagger}$ Corresponding author: Department of Mathematics, Zhengzhou University, Zhengzhou, P.R. China. E-mail: chyao@lsec.cc.ac.cn.

${ }_{\ddagger}^{\ddagger}$ Department of Mathematics, National University of Finance and Economics, Beijing, P.R China. E-mail:shjia@lsec.cc.ac.cn 


\section{Introduction}

Maxwell's equations describe the evolutions of the electromagnetic fields. Great interests have been shown on the study of Maxwell's equations by finite element methods, see e.g. [1, 4, 5, 8, 9, 10, 18, 19, 20, 22, 23, 24, 25].

Superconvergence of FEMs is a phenomenon that the convergence rate exceeds what general cases can provide, which can be achieved for smoother solutions with structured meshes. Many studies have been conducted for superconvergence analysis, see e.g. [7, 11, 16]. In 1994, Monk initiated the investigation on superconvergence for Maxwell's equations [21]. Recently, Lin and his collaborators systematically developed global superconvergence for the Maxwell's equations using the integral identity methods and postprocessing interpolations. All these works are for conforming finite elements, for example, ECHL element [13] and Nedelec element [12, 16, 29].

However, in the context of nonconforming mixed finite elements for the Maxwell's equations, to our best knowledge, there is no any result for global superconvergence, especially in three-dimensional case. A nonconforming mixed finite element approximation of time-harmonic Maxwell's equations with absorbing boundary condition was developed from the Rannacher-Turek nonconforming element by Douglas etc. in [6]. The $O\left(h^{\frac{1}{2}}\right)$ convergence rate for electric field $E$ and magnetic field $H$ in $L^{2}$-norm was presented. Unfortunately, the superclose and global superconvergence property cannot be achieved for this element, see the reasons in [14]. The Rannacher-Turek nonconforming element and its variational forms have been studied in many papers, see e.g. $[2,3,14,28,30]$. One of the variational forms of this element is the $E Q_{1}^{r o t}$ element. In [14], the superclose property was obtained for this $E Q_{1}^{\text {rot }}$ element for the elliptic problem based on the integral identity formulations. The authors also constructed a postprocess interpolation which resulted in a superconvergence of the order $O\left(h^{2}\right)$ in $H^{1}$-seminorm for the discretization error of the preprocessed discrete solution to the solution itself. In [17], asymptotic expansions of the nonconforming finite element $E Q_{1}^{\text {rot }}$ for the low-frequency time-harmonic Maxwell's equations were presented. Superclose and Superconvergence results of $O\left(h^{2}\right)$ error were obtained in the discrete $H($ curl) space.

In this paper, a nonconforming mixed finite element will be developed from the $E Q_{1}^{r o t}$ element for the three-dimensional time-harmonic Maxwell's equations. Superclose property can be demonstrated through asymptotic expansion method [13], which is much simpler than the integral identity method 
when analyzing the superclose properties for nonconforming finite elements. Superconvergence results can also be derived by a postprocessing procedure. Furthermore, extrapolation operators will be constructed to improve the accuracy based on the asymptotic expansion formulations.

This paper is organized as follows. In section 2, based on the mixed formulation of time-harmonic Maxwell's equations, a new nonconforming mixed finite element method is proposed. In section 3, asymptotic expansion behavior is demonstrated for interpolation error and nonconforming error of the nonconforming mixed finite element. In section 4, superclose and superconvergence analysis of electric field $E$ and magnetic field $H$ is derived, respectively. In section 5 , the accuracy is further improved by extrapolation method. To end this paper, we make some concluding remarks in section 6 .

\section{Time-Harmonic Maxwell's Equations and a Nonconforming Mixed Finite Element Ap- proximation}

Let $\Omega$ be a bounded cubic domain in $\mathcal{R}^{3}$ with boundary $\partial \Omega=\bigcup_{i=1}^{6} \partial \Omega_{i}$, where $\partial \Omega_{i}, i=1, \cdots, 6$ is the front, back, right, left, upper and lower face of $\Omega$, respectively, and denote the unit outward normal $\mathbf{n}$. Consider the following Maxwell's equations [6, 19]:

$$
\begin{array}{ll}
\sigma E-\operatorname{curl} H=F, & \text { in } \Omega, \\
i \omega \mu H+\operatorname{curl} E=0, & \text { in } \Omega, \\
E \times \mathbf{n}=0, & \text { on } \partial \Omega .
\end{array}
$$

where $\omega$ is a given angular frequency, and assume that $\sigma, \mu$ are constants. The existence and uniqueness results for the solution of equations (1)-(3) was given in [26].

A weak formulation of system (1)-(3) will be as follows: Find $(E, H) \in$ $\mathbf{H}_{\mathbf{0}}(\mathbf{c u r l}, \Omega) \times\left(L^{2}(\Omega)\right)^{3}$ such that

$$
\begin{array}{ll}
(\sigma E, \phi)-(H, \operatorname{curl} \phi)=(F, \phi), & \forall \phi \in \mathbf{H}_{\mathbf{0}}(\operatorname{curl}, \Omega), \\
(i \omega \mu H, \psi)+(\operatorname{curl} E, \psi)=0, & \forall \psi \in\left(L^{2}(\Omega)\right)^{3} .
\end{array}
$$


where

$$
\mathbf{H}_{\mathbf{0}}(\operatorname{curl}, \Omega)=\left\{\phi \in\left(L^{2}(\Omega)\right)^{3}\left|\operatorname{curl} \phi \in\left(L^{2}(\Omega)\right)^{3}, \phi \times \mathbf{n}\right|_{\partial \Omega}=0\right\} .
$$

Now we propose a new nonconforming mixed finite element. Let $\mathcal{J}_{h}$ be a uniform cube partition of domain $\Omega, 2 h_{x}, 2 h_{y}, 2 h_{z}$ is the length, width and height of element $\mathrm{K}, h_{K}=\max \left\{h_{x}, h_{y}, h_{z}\right\}_{K}, h=\max _{K}\left\{h_{K}\right\},\left(x_{K}, y_{K}, z_{K}\right)$ be the center of element $K$. Let $\hat{K}=[-1,1]^{3}$ be the reference element, then there exists an affine mapping $F_{K}: \hat{K} \longrightarrow K$

$$
\left\{\begin{array}{l}
x=x_{K}+h_{x} \hat{x} \\
y=y_{K}+h_{y} \hat{y} \\
z=z_{K}+h_{z} \hat{z}
\end{array}\right.
$$

Let $\hat{Q}=\hat{Q}_{x} \times \hat{Q}_{y} \times \hat{Q}_{z}$, where

$$
\begin{aligned}
& \hat{Q}_{x}=\operatorname{Span}\left\{1, \hat{y}, \hat{z}, \hat{y}^{2}, \hat{z}^{2}\right\}, \\
& \hat{Q}_{y}=\operatorname{Span}\left\{1, \hat{z}, \hat{x}, \hat{z}^{2}, \hat{x}^{2}\right\}, \\
& \hat{Q}_{z}=\operatorname{Span}\left\{1, \hat{x}, \hat{y}, \hat{x}^{2}, \hat{y}^{2}\right\} .
\end{aligned}
$$

Denote $\hat{g}_{i}, i=1, \cdots, 6$, be the front, back, right, left, upper and lower face of the reference element. For $\hat{\phi} \in \hat{Q}(\hat{K})$, the local interpolation operator on the reference element is defined as : $\hat{\pi}: \mathbf{H}_{\mathbf{0}}(\mathbf{c u r l}, \hat{K}) \longrightarrow \hat{Q}(\hat{K})$

$$
\frac{1}{\left|\hat{g}_{i}\right|} \int_{\hat{g}_{i}}(\hat{\pi} \hat{\phi}-\hat{\phi}) d \hat{s}=0, i=1,2, \cdots, 6, \frac{1}{|\hat{K}|} \int_{\hat{K}}(\hat{\pi} \hat{\phi}-\hat{\phi}) d \hat{x} d \hat{y} d \hat{z}=0 .
$$

In every element $K$, the local interpolation operator is defined as

$$
\pi_{K} \phi=(\hat{\pi} \hat{\phi}) \circ F_{K}^{-1},
$$

so the interpolation operator $\pi_{h}$ in the domain $\Omega$ is defined as

$$
\left.\pi_{h} \phi\right|_{K}=\pi_{K} \phi
$$

Note that (7) provides fifteen degrees of freedom needed to determine on an element in $\hat{Q}(\hat{K})$.

Next let $\hat{S}=\hat{S}_{x} \times \hat{S}_{y} \times \hat{S}_{z}$, where

$$
\begin{aligned}
& \hat{S}_{x}=\operatorname{Span}\{1, \hat{y}, \hat{z}\}, \\
& \hat{S}_{y}=\operatorname{Span}\{1, \hat{z}, \hat{x}\}, \\
& \hat{S}_{z}=\operatorname{Span}\{1, \hat{x}, \hat{y}\} .
\end{aligned}
$$


and define a local interpolation on the reference element $\hat{I}:\left(L^{2}(\hat{K})\right)^{3} \longrightarrow$ $\hat{S}(\hat{K})$ as: for $\hat{\psi}=\left(\hat{\psi}_{1}, \hat{\psi}_{2}, \hat{\psi}_{3}\right) \in \hat{S}(\hat{K})$

$$
\int_{\hat{K}}\left(\hat{I} \hat{\psi}_{l}-\hat{\psi}_{l}\right) d \hat{x} d \hat{y} d \hat{z}=0, \quad \int_{\hat{K}} \operatorname{curl}\left(\hat{I} \hat{\psi}_{l}-\hat{\psi}_{l}\right) d \hat{x} d \hat{y} d \hat{z}=0, l=1,2,3 .
$$

Here, curl in (8) is the two-dimensional defined as usual:

$$
\operatorname{curl} \hat{\psi}_{1}=\left(\frac{\partial \hat{\psi}_{1}}{\partial \hat{z}},-\frac{\partial \hat{\psi}_{1}}{\partial \hat{y}}\right), \quad \operatorname{curl} \hat{\psi}_{2}=\left(\frac{\partial \hat{\psi}_{2}}{\partial \hat{x}},-\frac{\partial \hat{\psi}_{2}}{\partial \hat{z}}\right), \quad \operatorname{curl} \hat{\psi}_{3}=\left(\frac{\partial \hat{\psi}_{3}}{\partial \hat{y}},-\frac{\partial \hat{\psi}_{3}}{\partial \hat{x}}\right) .
$$

In every element $K$, the local interpolation operator $I_{K}$ is defined as

$$
I_{K} \psi=(\hat{I} \hat{\psi}) \circ F_{K}^{-1},
$$

so the interpolation operator $I_{h}$ in the domain $\Omega$ is defined as

$$
\left.I_{h} \psi\right|_{K}=I_{K} \psi \text {. }
$$

Note that (8) provides nine degrees of freedom needed to determine on an element in $\hat{S}(\hat{K})$ and that

$$
\operatorname{curl} \hat{Q}=\hat{S} .
$$

The nonconforming mixed finite element space will be defined as

$$
\begin{aligned}
& V_{h}=\left\{\phi \in\left[L^{2}(\Omega)\right]^{3}:\left.\phi\right|_{K} \circ F_{K} \in \hat{Q}, K \in \mathcal{J}_{h}, \phi \times\left.\mathbf{n}\right|_{\partial \Omega}=0\right\}, \\
& W_{h}=\left\{\psi \in\left[L^{2}(\Omega)\right]^{3}:\left.\psi\right|_{K} \circ F_{K} \in \hat{S}, K \in \mathcal{J}_{h}\right\} .
\end{aligned}
$$

The discrete mixed finite element formulations of equation (4)-(5) show as follows: Find $\left(E_{h}, H_{h}\right) \in V_{h} \times W_{h}$ such that

$$
\begin{array}{ll}
\left(\sigma E_{h}, \phi\right)_{h}-\left(H_{h}, \operatorname{curl} \phi\right)_{h}=(F, \phi) & \forall \phi \in V_{h}, \\
\left(i \omega \mu H_{h}, \psi\right)_{h}+\left(\operatorname{curl} E_{h}, \psi\right)_{h}=0 & \forall \psi \in W_{h},
\end{array}
$$

with the discrete inner product in $\left(L^{2}(\Omega)\right)^{3}$ is defined by

$$
\left(u_{h}, v_{h}\right)_{h}=\sum_{K} \int_{K} u_{h} v_{h} d x d y d z, \quad \forall u_{h}, v_{h} \in V_{h} \text { or } W_{h} .
$$

and norm

$$
\left\|u_{h}\right\|_{0}=\left(\sum_{K} \int_{K} u_{h}^{2} d x d y d z\right)^{\frac{1}{2}}
$$


A traditional error estimation

$$
\left\|E-E_{h}\right\|_{0}+\left\|H-H_{h}\right\|_{0} \leq C h\left(\|E\|_{2}+\|H\|_{2}\right)
$$

can be derived with the boundary condition (3). The error estimation technique can be found in [27]. From the discussion in the next three sections, we can see that this new nonconforming mixed element has a global superconvergence property by constructing a postprocess operator which has an $O\left(h^{2}\right)$ accuracy, and has $O\left(h^{4}\right)$ accuracy by extrapolation method.

\section{Asymptotic Expansion Formulations}

In this section, the main aim is to derive asymptotic expansion formulations of interpolation error and nonconforming error.

$\forall \phi \in \mathbf{H}_{\mathbf{0}}(\mathbf{c u r l}, \Omega), \psi \in\left(L^{2}(\Omega)\right)^{3}$,

$$
D((E, H) ;(\phi, \psi)) \triangleq(\sigma E, \phi)-(H, \operatorname{curl} \phi)+(i \omega \mu H, \psi)+(\operatorname{curl} E, \psi),
$$

and $\forall \phi_{h} \in V_{h}, \psi_{h} \in W_{h}$,

$D_{h}\left(\left(E_{h}, H_{h}\right) ;\left(\phi_{h}, \psi_{h}\right)\right) \triangleq\left(\sigma E_{h}, \phi_{h}\right)_{h}-\left(H_{h}, \operatorname{curl} \phi_{h}\right)_{h}+\left(i \omega \mu H_{h}, \psi_{h}\right)_{h}+\left(\operatorname{curl} E_{h}, \psi_{h}\right)_{h}$.

From (4)-(5) and (9)-(10), $\forall \phi \in V_{h}, \psi \in W_{h}$, we have

$$
D_{h}\left(\left(E-E_{h}, H-H_{h}\right) ;(\phi, \psi)\right)=-<H, \phi \times \mathbf{n}>_{h},
$$

where $<H, \phi \times \mathbf{n}>_{h}=\sum_{K} \int_{\partial K} H \phi \times \mathbf{n} d s$. And

$$
\begin{aligned}
& D_{h}\left(\left(E_{h}-\pi_{h} E, H_{h}-I_{h} H\right) ;(\phi, \psi)\right) \\
& =<H, \phi \times \mathbf{n}>_{h}+D\left(\left(E-\pi_{h} E, H-I_{h} H\right) ;(\phi, \psi)\right) .
\end{aligned}
$$

Next, some useful asymptotic expansion formulations of (12) will be shown, and more details can be seen in [13]. Let $E=\left(E_{1}, E_{2}, E_{3}\right), H=$ $\left(H_{1}, H_{2}, H_{3}\right), \phi=\left(\phi_{1}, \phi_{2}, \phi_{3}\right), \psi=\left(\psi_{1}, \psi_{2}, \psi_{3}\right)$. For different $\hat{E}_{i}$ and $\hat{H}_{i}, i=$ $1,2,3$ on the reference element $\hat{K}$, their interpolations $\hat{\pi}_{\hat{K}} \hat{E}_{i}, \hat{I}_{\hat{K}} \hat{H}_{i}, i=1,2,3$ are shown in the following two tables respectively 


\begin{tabular}{|l|c|c|c|c|c|c|c|c|c|c|}
\hline$\hat{E}_{i}$ & 1 & $\hat{x}$ & $\hat{y}$ & $\hat{z}$ & $\hat{x} \hat{y}$ & $\hat{x} \hat{z}$ & $\hat{y} \hat{z}$ & $\hat{x}^{2}$ & $\hat{y}^{2}$ & $\hat{z}^{2}$ \\
\hline$\hat{\pi}_{\hat{K}} \hat{E}_{3}$ & 1 & $\hat{x}$ & $\hat{y}$ & 0 & 0 & 0 & 0 & $\hat{x}^{2}$ & $\hat{y}^{2}$ & $\frac{1}{3}$ \\
\hline$\hat{\pi}_{\hat{K}} \hat{E}_{2}$ & 1 & $\hat{x}$ & 0 & $\hat{z}$ & 0 & 0 & 0 & $\hat{x}^{2}$ & $\frac{1}{3}$ & $\hat{z}^{2}$ \\
\hline$\hat{\pi}_{\hat{K}} \hat{E}_{1}$ & 1 & 0 & $\hat{y}$ & $\hat{z}$ & 0 & 0 & 0 & $\frac{1}{3}$ & $\hat{y}^{2}$ & $\hat{z}^{2}$ \\
\hline$\hat{E}_{i}$ & $\hat{x}^{3}$ & $\hat{y}^{3}$ & $\hat{z}^{3}$ & $\hat{x}^{2} \hat{y}$ & $\hat{x} \hat{y}^{2}$ & $\hat{x}^{2} \hat{z}$ & $\hat{x} \hat{z}^{2}$ & $\hat{y}^{2} \hat{z}$ & $\hat{y} \hat{z}^{2}$ & $\hat{x} \hat{y} \hat{z}$ \\
\hline$\hat{\pi}_{\hat{K}} \hat{E}_{3}$ & $\hat{x}$ & $\hat{y}$ & 0 & $\frac{1}{3} \hat{y}$ & $\frac{1}{3} \hat{x}$ & 0 & $\frac{1}{3} \hat{x}$ & 0 & $\frac{1}{3} \hat{y}$ & 0 \\
\hline$\hat{\pi}_{\hat{K}} \hat{E}_{2}$ & $\hat{x}$ & 0 & $\hat{z}$ & 0 & $\frac{1}{3} \hat{x}$ & $\frac{1}{3} \hat{z}$ & $\frac{1}{3} \hat{x}$ & $\frac{1}{3} \hat{z}$ & 0 & 0 \\
\hline$\hat{\pi}_{\hat{K}} \hat{E}_{1}$ & 0 & $\hat{y}$ & $\hat{z}$ & $\frac{1}{3} \hat{y}$ & 0 & $\frac{1}{3} \hat{z}$ & 0 & $\frac{1}{3} \hat{z}$ & $\frac{1}{3} \hat{y}$ & 0 \\
\hline
\end{tabular}

\begin{tabular}{|l|c|c|c|c|c|c|c|c|c|c|}
\hline$\hat{H}_{i}$ & 1 & $\hat{x}$ & $\hat{y}$ & $\hat{z}$ & $\hat{x} \hat{y}$ & $\hat{x} \hat{z}$ & $\hat{y} \hat{z}$ & $\hat{x}^{2}$ & $\hat{y}^{2}$ & $\hat{z}^{2}$ \\
\hline$\hat{I}_{\hat{K}} \hat{H}_{3}$ & 1 & $\hat{x}$ & $\hat{y}$ & 0 & 0 & 0 & 0 & $\frac{1}{3}$ & $\frac{1}{3}$ & $\frac{1}{3}$ \\
\hline$\hat{I}_{\hat{K}} \hat{H}_{2}$ & 1 & $\hat{x}$ & 0 & $\hat{z}$ & 0 & 0 & 0 & $\frac{1}{3}$ & $\frac{1}{3}$ & $\frac{1}{3}$ \\
\hline$\hat{I}_{\hat{K}} \hat{H}_{1}$ & 1 & 0 & $\hat{y}$ & $\hat{z}$ & 0 & 0 & 0 & $\frac{1}{3}$ & $\frac{1}{3}$ & $\frac{1}{3}$ \\
\hline$\hat{H}_{i}$ & $\hat{x}^{3}$ & $\hat{y}^{3}$ & $\hat{z}^{3}$ & $\hat{x}^{2} \hat{y}$ & $\hat{x} \hat{y}^{2}$ & $\hat{x}^{2} \hat{z}$ & $\hat{x} \hat{z}^{2}$ & $\hat{y}^{2} \hat{z}$ & $\hat{y} \hat{z}^{2}$ & $\hat{x} \hat{y} \hat{z}$ \\
\hline$\hat{I}_{\hat{K}} \hat{H}_{3}$ & $\hat{x}$ & $\hat{y}$ & 0 & $\frac{1}{3} \hat{y}$ & $\frac{1}{3} \hat{x}$ & 0 & $\frac{1}{3} \hat{x}$ & 0 & $\frac{1}{3} \hat{y}$ & 0 \\
\hline$\hat{I}_{\hat{K}} \hat{H}_{2}$ & $\hat{x}$ & 0 & $\hat{z}$ & 0 & $\frac{1}{3} \hat{x}$ & $\frac{1}{3} \hat{z}$ & $\frac{1}{3} \hat{x}$ & $\frac{1}{3} \hat{z}$ & 0 & 0 \\
\hline$\hat{I}_{\hat{K}} \hat{H}_{1}$ & 0 & $\hat{y}$ & $\hat{z}$ & $\frac{1}{3} \hat{y}$ & 0 & $\frac{1}{3} \hat{z}$ & 0 & $\frac{1}{3} \hat{z}$ & $\frac{1}{3} \hat{y}$ & 0 \\
\hline
\end{tabular}

When

$$
h_{1} \equiv h_{x}, h_{2} \equiv h_{y}, h_{3} \equiv h_{z}, \forall K \in \mathcal{J}_{h},
$$

we call the mesh $\mathcal{J}_{h}$ is uniform [13].

Theorem 3.1. Assume that $E \in\left(H^{4}(\Omega)\right)^{3} \cap \mathbf{H}_{\mathbf{0}}(\mathbf{c u r l}, \Omega)$ and mesh $\mathcal{J}_{h}$ is uniform, we have

$$
\left(\sigma\left(E-\pi_{h} E\right), \phi\right)_{h}=O\left(h^{4}\right)\|E\|_{4}\|\phi\|_{0}, \quad \forall \phi \in V_{h} .
$$

Proof: From

$$
\begin{aligned}
& \left(\sigma\left(E-\pi_{h} E\right), \phi\right)_{h}=\sum_{K} \int_{K}\left(\sigma\left(E_{1}-\pi_{K} E_{1}\right) \cdot \phi_{1}\right. \\
& \left.+\sigma\left(E_{2}-\pi_{K} E_{2}\right) \cdot \phi_{2}+\sigma\left(E_{3}-\pi_{K} E_{3}\right) \cdot \phi_{3}\right) d x d y d z,
\end{aligned}
$$

Here we only consider one term. Let bilinear form

$$
B\left(\hat{E}_{1}, \hat{\phi}_{1}\right)=\int_{\hat{K}}\left(\hat{E}_{1}-\hat{\pi}_{\hat{K}} \hat{E}_{1}\right) \cdot \hat{\phi}_{1} d \hat{x} d \hat{y} d \hat{z}
$$


then we have

$$
\left|B\left(\hat{E}_{1}, \hat{\phi}_{1}\right)\right| \leq C\left\|\hat{E}_{1}\right\|_{4, \hat{K}}\left\|\hat{\phi}_{1}\right\|_{0, \hat{K}} .
$$

Here and after let $\hat{\phi}_{1}=\left(1, \hat{y}, \hat{z}, \hat{y}^{2}, \hat{z}^{2}\right)$. By calculating,

$$
\begin{aligned}
& B\left(\hat{y}^{3}, \hat{\phi}_{1}\right)=\int_{\hat{K}}\left(\hat{y}^{3}-\hat{y}\right)\left(1, \hat{y}, \hat{z}, \hat{y}^{2}, \hat{z}^{2}\right) d \hat{x} d \hat{y} d \hat{z} \\
& =-\frac{16}{15}(0,1,0,0,0)=-\frac{1}{45} \int_{\hat{K}} \hat{E}_{1 \hat{y} \hat{y} \hat{y}} \hat{\phi}_{1 \hat{y}} d \hat{x} d \hat{y} d \hat{z}, \quad \hat{E}_{1}=\hat{y}^{3}, \\
& B\left(\hat{z}^{3}, \hat{\phi}_{1}\right)=-\frac{1}{45} \int_{\hat{K}} \hat{E}_{1 \hat{z} \hat{z} \hat{z}} \hat{\phi}_{1 \hat{z}} d \hat{x} d \hat{y} d \hat{z}, \quad \hat{E}_{1}=\hat{z}^{3}, \\
& B\left(\hat{P}_{3} /\left\{\hat{y}^{3}, \hat{z}^{3}\right\}, \hat{\phi}_{1}\right)=0 .
\end{aligned}
$$

Let

$B\left(\hat{E}_{1}, \hat{\phi}_{1}\right)=-\frac{1}{45} \int_{\hat{K}} \hat{E}_{1 \hat{y} \hat{y} \hat{y}} \hat{\phi}_{1 \hat{y}} d \hat{x} d \hat{y} d \hat{z}-\frac{1}{45} \int_{\hat{K}} \hat{E}_{1 \hat{z} \hat{z} \hat{z}} \hat{\phi}_{1 \hat{z}} d \hat{x} d \hat{y} d \hat{z}+G\left(\hat{E}_{1}, \hat{\phi}_{1}\right)$,

we find

$$
G\left(\hat{P}_{3}, \hat{\phi}_{1}\right)=0 .
$$

Therefore, by Bramble-Hilbert Lemma [see Lemma 2.6, [13]], we have

$$
B\left(\hat{E}_{1}, \hat{\phi}_{1}\right)=-\frac{1}{45} \int_{\hat{K}}\left(\hat{E}_{1 \hat{y} \hat{y} \hat{y}} \hat{\phi}_{1 \hat{y}}+\hat{E}_{1 \hat{z} \hat{z} \hat{z}} \hat{\phi}_{1 \hat{z}}\right) d \hat{x} d \hat{y} d \hat{z}+O(1)\left|\hat{E}_{1}\right|_{4, \hat{K}}\left\|\hat{\phi}_{1}\right\|_{0, \hat{K}} .
$$

Hence, if the partition is uniform

$$
\begin{aligned}
& \int_{\Omega} \sigma\left(E_{1}-\pi_{K} E_{1}\right) \cdot \phi_{1} d x d y d z \\
& =\sigma \sum_{K}\left(-\frac{h_{y}^{4}}{45} \int_{K} E_{1 y y y} \phi_{1 y} d x d y d z-\frac{h_{z}^{4}}{45} \int_{K} E_{1 z z z} \phi_{1 z} d x d y d z\right)+O\left(h^{4}\right)\left|E_{1}\right|_{4}\left\|\phi_{1}\right\|_{0} \\
& =\sigma\left(\frac{h_{y}^{4}}{45} \int_{\Omega} E_{1 y y y y} \phi_{1} d x d y d z+\frac{h_{z}^{4}}{45} \int_{\Omega} E_{1 z z z z} \phi_{1} d x d y d z\right)+O\left(h^{4}\right)\left|E_{1}\right|_{4}\left\|\phi_{1}\right\|_{0} .
\end{aligned}
$$

Here we use integration by parts and the continuity of $\phi_{1}$ on the interface of two adjoint element. We also need the boundary condition $E \times \mathbf{n}=0$, which implies $E_{1}, E_{3}$ equal to 0 on $\partial \Omega_{1}, \partial \Omega_{2}, E_{3}, E_{2}$ equal to 0 on $\partial \Omega_{3}, \partial \Omega_{4}$, and $E_{2}, E_{1}$ equal to 0 on $\partial \Omega_{5}, \partial \Omega_{6}$. That means that all the tangential directional of $E_{i}$ equal to 0 on the corresponding faces. 
Similarly,

$$
\begin{aligned}
& \int_{\Omega} \sigma\left(E_{2}-\pi_{K} E_{2}\right) \cdot \phi_{2} d x d y d z \\
& =\sigma\left(\frac{h_{x}^{4}}{45} \int_{\Omega} E_{2 x x x x} \phi_{2} d x d y d z+\frac{h_{z}^{4}}{45} \int_{\Omega} E_{2 z z z z} \phi_{2} d x d y d z\right) \\
& +O\left(h^{4}\right)\left|E_{2}\right|_{4}\left\|\phi_{2}\right\|_{0}, \\
& \int_{\Omega} \sigma\left(E_{3}-\pi_{K} E_{3}\right) \cdot \phi_{3} d x d y d z \\
& =\sigma\left(\frac{h_{x}^{4}}{45} \int_{\Omega} E_{3 x x x x} \phi_{3} d x d y d z+\frac{h_{y}^{4}}{45} \int_{\Omega} E_{3 y y y y} \phi_{3} d x d y d z\right) \\
& +O\left(h^{4}\right)\left|E_{3}\right|_{4}\left\|\phi_{3}\right\|_{0} .
\end{aligned}
$$

Combining (15)-(17), we can finish the proof.

Theorem 3.2. Assume that $H \in\left(H^{4}(\Omega)\right)^{3}$ and mesh $\mathcal{J}_{h}$ is uniform, we have

$$
\left(i \omega \mu\left(H-I_{h} H\right), \psi\right)_{h}=O\left(h^{4}\right)\|H\|_{4}\|\psi\|_{1 h}, \quad \forall \psi \in W_{h} .
$$

where $\|\psi\|_{1 h}=\sum_{K} \sum_{|\alpha| \leq 1}\left(\int_{K}\left(D^{\alpha} \psi\right)^{2}\right)^{1 / 2}$.

\section{Proof:}

Now we consider the asymptotic expansion for

$$
\begin{aligned}
& \left(i \omega \mu\left(H-I_{K} H\right), \psi\right)_{h}=\sum_{K} \int_{K}\left(i \omega \mu\left(H_{1}-I_{K} H_{1}\right) \cdot \psi_{1}\right. \\
& \left.+i \omega \mu\left(H_{2}-I_{K} H_{2}\right) \cdot \psi_{2}+i \omega \mu\left(H_{3}-I_{K} H_{3}\right) \cdot \psi_{3}\right) d x d y d z .
\end{aligned}
$$

Here and after denote $\hat{\psi}_{3}=(1, \hat{x}, \hat{y})$. Let bilinear form

$$
B\left(\hat{H}_{3}, \hat{\psi}_{3}\right)=\int_{\hat{K}}\left(\hat{H}_{3}-\hat{I}_{\hat{K}} \hat{H}_{3}\right) \cdot \hat{\psi}_{3} d \hat{x} d \hat{y} d \hat{z}
$$

Then we have

$$
\left|B\left(\hat{H}_{3}, \hat{\psi}_{3}\right)\right| \leq C\left\|\hat{E}_{3}\right\|_{4, \hat{K}}\left\|\hat{\psi}_{3}\right\|_{0, \hat{K}}
$$


By calculating

$$
\begin{aligned}
& B\left(\hat{x}^{3}, \hat{\psi}_{3}\right)=\int_{\hat{K}}\left(\hat{x}^{3}-\hat{x}\right)(1, \hat{x}, \hat{y}) d \hat{x} d \hat{y} d \hat{z} \\
& =-\frac{16}{15}(0,1,0)=-\frac{1}{45} \int_{\hat{K}} \hat{H}_{3 \hat{x} \hat{x} \hat{x}} \hat{\psi}_{3 \hat{x}} d \hat{x} d \hat{y} d \hat{z}, \quad \hat{H}_{3}=\hat{x}^{3}, \\
& B\left(\hat{y}^{3}, \hat{\psi}_{3}\right)=-\frac{1}{45} \int_{\hat{K}} \hat{H}_{3 \hat{y} \hat{y} \hat{y}} \hat{\psi}_{3 \hat{y}} d \hat{x} d \hat{y} d \hat{z}, \quad \hat{H}_{3}=\hat{y}^{3}, \\
& B\left(\hat{P}_{3} /\left\{\hat{x}^{3}, \hat{y}^{3}\right\}, \hat{\psi}_{3}\right)=0 .
\end{aligned}
$$

Let

$$
B\left(\hat{H}_{3}, \hat{\psi}_{3}\right)=-\frac{1}{45} \int_{\hat{K}}\left(\hat{H}_{3 \hat{x} \hat{x} \hat{x}} \hat{\psi}_{3 \hat{x}}+\hat{H}_{3 \hat{y} \hat{y} \hat{y}} \hat{\psi}_{3 \hat{y}}\right) d \hat{x} d \hat{y} d \hat{z}+G_{3}\left(\hat{H}_{3}, \hat{\psi}_{3}\right)
$$

We have

$$
G_{3}\left(\hat{P}_{3}, \hat{\psi}_{3}\right)=0
$$

Therefore,

$$
B\left(\hat{H}_{3}, \hat{\psi}_{3}\right)=-\frac{1}{45} \int_{\hat{K}}\left(\hat{H}_{3 \hat{x} \hat{x} \hat{x}} \hat{\psi}_{3 \hat{x}}+\hat{H}_{3 \hat{y} \hat{y} \hat{y}} \hat{\psi}_{3 \hat{y}}\right) d \hat{x} d \hat{y} d \hat{z}+O(1)\left|\hat{H}_{3}\right|_{4, \hat{K}}\left\|\hat{\psi}_{3}\right\|_{0, \hat{K}}
$$

Hence,

$$
\begin{aligned}
& \int_{\Omega} i \omega \mu\left(H_{3}-I_{h} H_{3}\right) \psi_{3} d x d y d z \\
& =i \omega \mu \sum_{K}\left(-\frac{h_{x}^{4}}{45} \int_{K} H_{3 x x x} \psi_{3 x} d x d y d z-\frac{h_{y}^{4}}{45} \int_{K} H_{3 y y y} \psi_{3 y} d x d y d z\right) \\
& +O\left(h^{4}\right)\left|H_{3}\right|_{4}\left\|\psi_{3}\right\|_{0} .
\end{aligned}
$$

Similarly,

$$
\begin{aligned}
& \int_{\Omega} i \omega \mu\left(H_{2}-I_{h} H_{2}\right) \psi_{2} d x d y d z \\
& =i \omega \mu \sum_{K}\left(-\frac{h_{x}^{4}}{45} \int_{K} H_{2 x x x} \psi_{2 x} d x d y d z-\frac{h_{z}^{4}}{45} \int_{K} H_{2 z z z} \psi_{2 z} d x d y d z\right) \\
& +O\left(h^{4}\right)\left|H_{2}\right|_{4}\left\|\psi_{2}\right\|_{0},
\end{aligned}
$$




$$
\begin{aligned}
& \int_{\Omega} i \omega \mu\left(H_{1}-I_{h} H_{1}\right) \psi_{1} d x d y d z \\
& =i \omega \mu \sum_{K}\left(-\frac{h_{y}^{4}}{45} \int_{K} H_{1 y y y} \psi_{1 y} d x d y d z-\frac{h_{z}^{4}}{45} \int_{K} H_{1 z z z} \psi_{1 z} d x d y d z\right) \\
& +O\left(h^{4}\right)\left|H_{1}\right|_{4}\left\|\psi_{1}\right\|_{0} .
\end{aligned}
$$

For (19)-(21), using Cauchy-Schwartz inequality, we can get (18). Now we can finish the proof.

Theorem 3.3. Assume that $H \in\left(H^{4}(\Omega)\right)^{3}$ and mesh $\mathcal{J}_{h}$ is uniform, we have

$$
\left(\left(H-I_{h} H\right), \operatorname{curl} \phi\right)_{h}=O\left(h^{4}\right)\|H\|_{4}\|\phi\|_{2 h}, \quad \forall \phi \in V_{h} .
$$

where $\|\phi\|_{2 h}=\sum_{K} \sum_{|\alpha| \leq 2}\left(\int_{K}\left(D^{\alpha} \phi\right)^{2}\right)^{1 / 2}$.

Proof: From

$$
\begin{aligned}
& \left(\left(H-I_{K} H\right), \operatorname{curl} \phi\right)_{h}=\sum_{K} \int_{K}\left[\left(H_{1}-I_{h} H_{1}\right)\left(\phi_{3 y}-\phi_{2 z}\right)\right. \\
& \left.-\left(H_{2}-I_{h} H_{2}\right)\left(\phi_{3 x}-\phi_{1 z}\right)+\left(H_{3}-I_{h} H_{3}\right)\left(\phi_{2 x}-\phi_{1 y}\right)\right] d x d y d z .
\end{aligned}
$$

Only consider the first term $\int_{K}\left(H_{1}-I_{h} H_{1}\right) \phi_{3 y} d x d y d z$. Here and after, denote

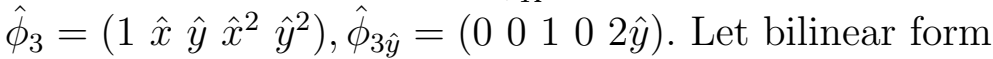

$$
B\left(\hat{H}_{1}, \hat{\phi}_{3}\right)=\int_{\hat{K}}\left(\hat{H}_{1}-\hat{I}_{\hat{K}} \hat{H}_{1}\right) \hat{\phi}_{3 \hat{y}} d \hat{x} d \hat{y} d \hat{z}
$$

then

$$
\left|B\left(\hat{H}_{1}, \hat{\phi}_{3}\right)\right| \leq C\left\|\hat{H}_{1}\right\|_{4}\left\|\hat{\phi}_{3}\right\|_{0} .
$$

By calculating,

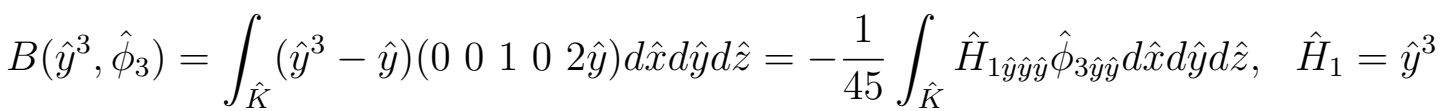

$$
\begin{aligned}
& B\left(\hat{P}_{3} /\left\{\hat{y}^{3}\right\}, \hat{\phi}_{3}\right)=0 \text {. }
\end{aligned}
$$

Let

$$
B\left(\hat{y}^{3}, \hat{\phi}_{3}\right)=-\frac{1}{45} \int_{\hat{K}} \hat{H}_{1 \hat{y} \hat{y} \hat{y}} \hat{\phi}_{3 \hat{y} \hat{y}} d \hat{x} d \hat{y} d \hat{z}+G\left(\hat{H}_{1}, \hat{\phi}_{3}\right)
$$


then

$$
G\left(\hat{P}_{3}, \hat{\phi}_{3}\right)=0
$$

Therefore,

$$
B\left(\hat{H}_{1}, \hat{\phi}_{3}\right)=-\frac{1}{45} \int_{\hat{K}} \hat{H}_{1 \hat{y} \hat{y} \hat{y}} \hat{\phi}_{3 \hat{y} \hat{y}} d \hat{x} d \hat{y} d \hat{z}+O(1)\left|\hat{H}_{1}\right|_{4, \hat{K}}\left\|\hat{\phi}_{3}\right\|_{0, \hat{K}} .
$$

Hence, integration by parts and if the partition is uniform, we have

$$
\begin{aligned}
& \int_{\Omega}\left(H_{1}-I_{h} H_{1}\right) \phi_{3 y} d x d y d z=\sum_{K} \int_{K}\left(H_{1}-I_{K} H_{1}\right) \phi_{3 y} d x d y d z \\
& =\sum_{K}-\frac{h_{y}^{4}}{45} \int_{K} H_{1 y y y} \phi_{3 y y} d x d y d z+O\left(h^{4}\right)\left|H_{1}\right|_{4}\left\|\phi_{3}\right\|_{0} . \\
& =O\left(h^{4}\right)\left\|H_{1}\right\|_{4}\left\|\phi_{3}\right\|_{2 h}
\end{aligned}
$$

Similarly,

$$
\begin{aligned}
& \int_{\Omega}-\left(H_{1}-I_{h} H_{1}\right) \phi_{2 z} d x d y d z=O\left(h^{4}\right)\left|H_{1}\right|_{4}\left\|\phi_{2}\right\|_{2 h}, \\
& \int_{\Omega}-\left(H_{2}-I_{h} H_{2}\right) \phi_{3 x} d x d y d z=O\left(h^{4}\right)\left|H_{2}\right|_{4}\left\|\phi_{3}\right\|_{2 h}, \\
& \int_{\Omega}\left(H_{2}-I_{h} H_{2}\right) \phi_{1 z} d x d y d z=O\left(h^{4}\right)\left|H_{2}\right|_{4}\left\|\phi_{1}\right\|_{2 h}, \\
& \int_{\Omega}\left(H_{3}-I_{h} H_{3}\right) \phi_{2 x} d x d y d z=O\left(h^{4}\right)\left|H_{3}\right|_{4}\left\|\phi_{2}\right\|_{2 h}, \\
& \int_{\Omega}-\left(H_{3}-I_{h} H_{3}\right) \phi_{1 y} d x d y d z=O\left(h^{4}\right)\left|H_{3}\right|_{4}\left\|\phi_{1}\right\|_{2 h} .
\end{aligned}
$$

Combining (23)-(28), we can finish the proof.

Theorem 3.4. Assume that $E \in \mathbf{H}_{0}(\mathbf{c u r l}, \Omega)$, we have

$$
\left(\operatorname{curl}\left(E-\pi_{h} E\right), \psi\right)_{h}=0, \quad \forall \psi \in W_{h} .
$$

Proof: By the definition of interpolation operator $\pi_{h}$ and $\operatorname{curl} \psi \in$ $\operatorname{span}\{1\}$, we have

$\left(\operatorname{curl}\left(E-\pi_{h} E\right), \psi\right)_{h}=\left(\left(E-\pi_{h} E\right), \operatorname{curl} \psi\right)_{h}+<\left(E-\pi_{h} E\right) \times \mathbf{n}, \psi>_{h}=0$. 
Theorem 3.5. Assume that $H \in\left(H^{5}(\Omega)\right)^{3}$ and mesh $\mathcal{J}_{h}$ is uniform, we have the nonconforming error

$$
\begin{aligned}
<H, \phi \times \mathbf{n}>_{h} \quad & =\frac{h_{x}^{2}}{3} \int_{\Omega}\left(H_{1 x x y} \phi_{3}-H_{1 x x z} \phi_{2}\right) d x d y d z \\
& +\frac{h_{y}^{2}}{3} \int_{\Omega}\left(H_{2 y y z} \phi_{1}-H_{2 x y y} \phi_{3}\right) d x d y d z \\
& +\frac{h_{z}^{2}}{3} \int_{\Omega}\left(H_{3 x z z} \phi_{2}-H_{3 y z z} \phi_{1}\right) d x d y d z \\
& +O\left(h^{4}\right)\|H\|_{5}\|\phi\|_{2 h}, \quad \forall \phi \in V_{h} .
\end{aligned}
$$

Proof: Let

$$
\begin{aligned}
& <H, \phi \times \mathbf{n}>_{h}=\sum_{K} \int_{\partial K} H \cdot \phi \times \mathbf{n} d s \\
& =\sum_{K}\left[\left(\int_{g_{2}}-\int_{g_{1}}\right)\left(H_{3} \phi_{1}-H_{1} \phi_{3}\right) d x d z+\left(\int_{g_{4}}-\int_{g_{3}}\right)\left(H_{2} \phi_{3}-H_{3} \phi_{2}\right) d y d z\right. \\
& \left.+\left(\int_{g_{5}}-\int_{g_{6}}\right)\left(H_{1} \phi_{2}-H_{2} \phi_{1}\right) d x d y\right] .
\end{aligned}
$$

Our task is to show that the summation of face-integrate is of high-order, and even has an error expansion. The technique is to approximate the integrate $\phi_{i}, i=1,2,3$ on each face $g_{j}, j=1,2, \cdots, 6$ by its conforming part, average $\left.\bar{\phi}_{i}\right|_{g_{j}}=\frac{1}{\left|g_{j}\right|} \int_{g_{j}} \phi_{i} d s$, which has continuity between elements, and we can also utilize the boundary condition $\phi \times \mathbf{n}=0$ on $\partial \Omega$. Hence, the summation

$$
\begin{aligned}
& \sum_{K}\left[\left(\int_{g_{2}}-\int_{g_{1}}\right)\left(H_{3} \bar{\phi}_{1}-H_{1} \bar{\phi}_{3}\right) d x d z+\left(\int_{g_{4}}-\int_{g_{3}}\right)\left(H_{2} \bar{\phi}_{3}-H_{3} \bar{\phi}_{2}\right) d y d z\right. \\
& \left.+\left(\int_{g_{5}}-\int_{g_{6}}\right)\left(H_{1} \bar{\phi}_{2}-H_{2} \bar{\phi}_{1}\right) d x d y\right]=0 .
\end{aligned}
$$

For convenience, we only consider one term, and the other is similar.

$$
\sum_{K}\left(\int_{g_{2}}-\int_{g_{1}}\right) H_{3} \phi_{1} d x d z=\sum_{K}\left(\int_{g_{2}}-\int_{g_{1}}\right) H_{3}\left(\phi_{1}-\bar{\phi}_{1}\right) d x d z .
$$

By the expansion [13]

$$
\left.\left(\phi_{1}-\bar{\phi}_{1}\right)\right|_{g_{j}}=\left(z-z_{K}\right) \phi_{1 z}-\left(\left(z-z_{K}\right)^{2}+\frac{h_{z}^{2}}{3}\right) \frac{\phi_{1 z z}}{2}, \quad j=1,2,
$$


we have

$$
\begin{aligned}
& \left(\int_{g_{2}}-\int_{g_{1}}\right) H_{3}\left(\phi_{1}-\bar{\phi}_{1}\right) \\
& =\left(\int_{g_{2}}-\int_{g_{1}}\right) H_{3}\left(\left(z-z_{K}\right) \phi_{1 z}-\left(\left(z-z_{K}\right)^{2}+\frac{h_{z}^{2}}{3}\right) \frac{\phi_{1 z z}}{2}\right) d x d z \\
& =\int_{K}\left[H_{3}\left(\left(z-z_{K}\right) \phi_{1 z}-\left(\left(z-z_{K}\right)^{2}+\frac{h_{z}^{2}}{3}\right) \frac{\phi_{1 z z}}{2}\right)\right]_{y} d y d x d z \\
& =\int_{K} H_{3 y}\left(\left(z-z_{K}\right) \phi_{1 z}-\left(\left(z-z_{K}\right)^{2}+\frac{h_{z}^{2}}{3}\right) \frac{\phi_{1 z z}}{2}\right) d x d y d z \\
& =\frac{h_{z}^{2}}{3} \int_{K} H_{3 y z} \phi_{1 z} d x d y d z-\frac{4 h_{z}^{4}}{45} \int_{K} H_{3 y z z} \phi_{1 z z} d x d y d z+O\left(h^{4}\right)\left|H_{3}\right|_{5, K}\left|\phi_{1}\right|_{0, K} . \\
& =\frac{h_{z}^{2}}{3} \int_{K} H_{3 y z} \phi_{1 z} d x d y d z+O\left(h^{4}\right)\left\|H_{3}\right\|_{5, K}\left|\phi_{1}\right|_{2, K} .
\end{aligned}
$$

Integrating by parts, we have

$$
\begin{aligned}
& \sum_{K}\left[\left(\int_{g_{2}}-\int_{g_{1}}\right) H_{3}\left(\phi_{1}-\bar{\phi}_{1}\right)\right] \\
& =\sum_{K}\left[-\frac{h_{z}^{2}}{3} \int_{K} H_{3 y z z} \phi_{1} d x d y d z+O\left(h^{4}\right)\|H\|_{5, K}|\phi|_{2, K}\right] .
\end{aligned}
$$

Similarly,

$$
\begin{aligned}
& \sum_{K}\left[\left(\int_{g_{2}}-\int_{g_{1}}\right)\left(-H_{1}\left(\phi_{3}-\bar{\phi}_{3}\right)\right) d x d z\right] \\
& =\sum_{K}\left[\frac{h_{x}^{2}}{3} \int_{K} H_{1 x x y} \phi_{3} d x d y d z+O\left(h^{4}\right)\|H\|_{5, K}|\phi|_{2, K}\right] .
\end{aligned}
$$

Here we need the boundary condition $\phi \times \mathbf{n}=0$ on $\partial \Omega$.

Therefore,

$$
\begin{aligned}
& \sum_{K}\left[\left(\int_{g_{2}}-\int_{g_{1}}\right)\left(\left(H_{3} \phi_{1}-H_{1} \phi_{3}\right)\right) d x d z\right] \\
& =\sum_{K}\left[-\frac{h_{z}^{2}}{3} \int_{K} H_{3 y z z} \phi_{1} d x d y d z\right. \\
& \left.+\frac{h_{x}^{2}}{3} \int_{K} H_{1 x x y} \phi_{3} d x d y d z+O\left(h^{4}\right)\|H\|_{5, K}|\phi|_{2, K}\right] .
\end{aligned}
$$


Similarly,

$$
\begin{aligned}
& \sum_{K}\left[\left(\int_{g_{4}}-\int_{g_{3}}\right)\left(H_{2} \phi_{3}-H_{3} \phi_{2}\right) d y d z\right] \\
& =\sum_{K}\left[-\frac{h_{2}^{2}}{3} \int_{K} H_{2 x y y} \phi_{3} d x d y d z+\frac{h_{3}^{2}}{3} \int_{K} H_{3 x z z} \phi_{2} d x d y d z\right. \\
& \left.+O\left(h^{4}\right)\|H\|_{5, K}|\phi|_{2, K}\right], \\
& \sum_{K}\left[\left(\int_{g_{5}}-\int_{g_{6}}\right)\left(H_{1} \phi_{2}-H_{2} \phi_{1}\right) d x d y\right] \\
& =\sum_{K}\left[-\frac{h_{1}^{2}}{3} \int_{K} H_{1 x x z} \phi_{2} d x d y d z+\frac{h_{2}^{2}}{3} \int_{K} H_{2 y y z} \phi_{1} d x d y d z\right. \\
& \left.+O\left(h^{4}\right)\|H\|_{5, K}|\phi|_{2, K}\right] .
\end{aligned}
$$

When the mesh $\mathcal{J}_{h}$ is uniform, combining (31)-(33), we can end the proof.

\section{Superclose and Superconvergence Analysis}

Now based on the theorems in Section 3, we can get the principal superclose result in this paper.

Theorem 4.1. Let $(E, H)$ and $\left(E_{h}, H_{h}\right)$ be the solution of (4)-(5) and (9)(10), respectively, $\pi_{h} E \in V_{h}$ and $I_{h} H \in W_{h}$ be the interpolations of $E$ and $H$, respectively. Assume $(E, H) \in\left(\mathbf{H}_{\mathbf{0}}(\mathbf{c u r l}, \Omega) \cap\left(H^{3}(\Omega)\right)^{3}\right) \times\left(H^{3}(\Omega)\right)^{3}$ and the mesh $\mathcal{J}_{h}$ is uniform, we have

$$
\left\|E_{h}-\pi_{h} E\right\|_{0}+\left\|H_{h}-I_{h} H\right\|_{0} \leq C h^{2}\left(\|E\|_{3}+\|H\|_{3}\right) .
$$

Proof: $\forall \phi \in \mathbf{H}_{\mathbf{0}}(\mathbf{c u r l}, \Omega), \psi \in\left(L^{2}(\Omega)\right)^{3}, \phi_{h} \in V_{h}, \psi_{h} \in W_{h}$, from the definition of $D((E, H) ;(\phi, \psi)), D_{h}\left(\left(E_{h}, H_{h}\right) ;\left(\phi_{h}, \psi_{h}\right)\right)$, and Theorem 3.1Theorem 3.5, we can get

$$
\begin{aligned}
& \left\|E_{h}-\pi_{h} E\right\|_{0}^{2}+\left\|H_{h}-I_{h} H\right\|_{0}^{2} \\
& \leq\left|D_{h}\left(\left(E_{h}-\pi_{h} E, H_{h}-I_{h} H\right),\left(E_{h}-\pi_{h} E, H_{h}-I_{h} H\right)\right)\right| \\
& \leq\left|<H,\left(E_{h}-\pi_{h} E\right) \times \mathbf{n}>_{h}\right| \\
& +\left|D_{h}\left(\left(E-\pi_{h} E, H-I_{h} H\right),\left(E-\pi_{h} E, H-I_{h} H\right)\right)\right| \\
& =O\left(h^{2}\right)\left(\|E\|_{3}+\|H\|_{3}\right)\left(\left\|E_{h}-\pi_{h} E\right\|_{0}+\left\|H_{h}-I_{h} H\right\|_{0}\right) .
\end{aligned}
$$


Then (34) follows.

Based on Theorem 4.1 and theory of constructing post-processing operator in $[13,15]$, there exists a post-processing operator $\Pi_{2 h}^{1}$ such that

$$
\begin{aligned}
& \text { 1. } \Pi_{2 h}^{1} \phi \in Q_{111}(\tau), \forall \phi \in\left(L^{2}(\Omega)\right)^{3}, \\
& \text { 2. }\left\|\Pi_{2 h}^{1} \phi-\phi\right\|_{0, \tau} \leq C h^{2}\|\phi\|_{2, \tau}, \quad \forall \phi \in\left(L^{2}(\Omega)\right)^{3}, \\
& \text { 3. }\left\|\Pi_{2 h}^{1} \phi\right\|_{0, \tau} \leq C\|\phi\|_{0, \tau}, \quad \forall \phi \in V_{h}, \\
& \text { 4. } \Pi_{2 h}^{1} \pi_{h} \phi=\Pi_{2 h}^{1} \phi, \quad \forall \phi \in\left(L^{2}(\Omega)\right)^{3},
\end{aligned}
$$

where $\tau$ can be constructed by merging the adjacent 8 elements into a big element.

The postprocessing operator $\Pi_{2 h}^{1}$ can be defined by the following procedure. Let $\tau \in \mathcal{J}_{2 h}$ consist of $2 \times 2 \times 2$ elements $K_{i} \in \mathcal{J}_{h}, i=1,2 \ldots 8 . V_{h}(\tau)$ denotes the space of nonconforming finite element functions of $V_{h}$ restricted onto $\tau$. We define the local interpolation operator $\Pi_{2 h}^{1}: V_{h}(\tau) \longrightarrow Q_{111}(\tau)$ by

$$
\int_{K_{i}}\left(\Pi_{2 h}^{1} E-E\right) d x d y d z=0, i=1,2, \cdots, 8
$$

It can be shown that the interpolation operator $\Pi_{2 h}^{1}$ is uniquely defined.

Here, we can also construct a post-processing operator $I_{2 h}^{1}$ concerning electric fields $H$, such that $I_{2 h}^{1} H \in Q_{111}(\tau)$ with properties (35)-(38) replacing $V_{h}$ with $W_{h}$ in (37).

$$
\int_{K_{i}}\left(I_{2 h}^{1} H-H\right) d x d y d z=0, i=1,2, \cdots, 8 .
$$

Therefore, we have the superconvergence result:

Theorem 4.2. Let $(E, H)$ and $\left(E_{h}, H_{h}\right)$ be the solution of (4)-(5) and (9)(10), respectively. Assume $(E, H) \in\left(\mathbf{H}_{\mathbf{0}}(\mathbf{c u r l}, \Omega) \cap\left(H^{3}(\Omega)\right)^{3}\right) \times\left(H^{3}(\Omega)\right)^{3}$ and the mesh $\mathcal{J}_{h}$ is uniform, we have

$$
\left\|E-\Pi_{2 h}^{1} E_{h}\right\|_{0}+\left\|H-I_{2 h}^{1} H_{h}\right\|_{0} \leq C h^{2}\left(\|E\|_{3}+\|H\|_{3}\right) .
$$

Proof: By (38)

$$
I_{2 h}^{1} H_{h}-H=I_{2 h}^{1} H_{h}-I_{2 h}^{1} I_{h} H+I_{2 h}^{1} I_{h} H-H .
$$


By (36)(37) and Theorem $\mathbf{3 . 2}$

$$
\left\|I_{2 h}^{1} H_{h}-H\right\|_{0} \leq C\left\|H_{h}-I_{h} H\right\|_{0}+C h^{2}\|H\|_{2}=O\left(h^{2}\right)\|H\|_{3} .
$$

Similarly,

$$
\left\|\Pi_{2 h}^{1} E_{h}-E\right\|_{0}=O\left(h^{2}\right)\|E\|_{3} .
$$

Hence, (39) follows.

\section{Extrapolation}

Extrapolation is also viewed as a superconvergence technique. In this section, we will discuss an extrapolation of the preprocessed discrete solution. In order to obtain asymptotic error expansion, we need to construct the following auxiliary equations:

Find $(\tilde{E}, \tilde{H}) \in \mathbf{H}_{\mathbf{0}}(\mathbf{c u r l}, \Omega) \times\left(L^{2}(\Omega)\right)^{3}$ such that

$$
\begin{array}{ll}
(\sigma \tilde{E}, \phi)-(\tilde{H}, \operatorname{curl} \phi)=S_{h}(\phi), & \forall \phi \in \mathbf{H}_{\mathbf{0}}(\operatorname{curl}, \Omega), \\
(i \omega \mu \tilde{H}, \psi)+(\operatorname{curl} \tilde{E}, \psi)=0, & \forall \psi \in\left(L^{2}(\Omega)\right)^{3},
\end{array}
$$

with the regularity property $[19]$ of the solution $(\tilde{E}, \tilde{H})$ :

$$
\|\tilde{E}\|_{2}+\|\tilde{H}\|_{2} \leq C\left(\|E\|_{3}+\|H\|_{3}\right) \text {. }
$$

where

$$
\begin{aligned}
& S_{h}(\phi)=\frac{h_{x}^{2}}{3 h^{2}} \int_{\Omega}\left(H_{1 x x y} \phi_{3}-H_{1 x x z} \phi_{2}\right) d x d y d z \\
& +\frac{h_{y}^{2}}{3 h^{2}} \int_{\Omega}\left(H_{2 y y z} \phi_{1}-H_{2 x y y} \phi_{3}\right) d x d y d z \\
& +\frac{h_{z}^{2}}{3 h^{2}} \int_{\Omega}\left(H_{3 x z z} \phi_{2}-H_{3 y z z} \phi_{1}\right) d x d y d z
\end{aligned}
$$

Obviously, $S_{h}$ has the following property

$$
S_{h}=S_{\frac{h}{2}}
$$

Let $\left(\tilde{E}_{h}, \tilde{H}_{h}\right) \in V_{h} \times W_{h}$ be the finite element solution approximation of $(\tilde{E}, \tilde{H})$, i.e.,

$$
\begin{array}{cc}
\left(\sigma \tilde{E}_{h}, \phi\right)_{h}-\left(\tilde{H}_{h}, \operatorname{curl} \phi\right)_{h}=S_{h}(\phi), & \forall \phi \in V_{h}, \\
\left(i \omega \mu \tilde{H}_{h}, \psi\right)_{h}+\left(\operatorname{curl} \tilde{E}_{h}, \psi\right)_{h}=0, & \forall \psi \in W_{h} .
\end{array}
$$


Theorem 5.1. Assume $(E, H) \in\left(\mathbf{H}_{\mathbf{0}}(\mathbf{c u r l}, \Omega) \cap\left(H^{4}(\Omega)\right)^{3}\right) \times\left(H^{5}(\Omega)\right)^{3}$, $\left(\pi_{h} E, I_{h} H\right)$ are the interpolation of $(E, H),\left(E_{h}, H_{h}\right)$ and $\left(\tilde{E}_{h}, \tilde{H}_{h}\right)$ are the finite element approximations of $(E, H)$ and $(\tilde{E}, \tilde{H})$, respectively. Then we have the following estimations

$$
\begin{aligned}
& \left\|E_{h}-\pi_{h} E-h^{2} \tilde{E}_{h}\right\|_{0}=O\left(h^{4}\right)\left(\|E\|_{4}+\|H\|_{5}\right), \\
& \left\|H_{h}-I_{h} H-h^{2} \tilde{H}_{h}\right\|_{0}=O\left(h^{4}\right)\left(\|E\|_{4}+\|H\|_{5}\right) .
\end{aligned}
$$

Proof: From Theorem 3.1- 3.5 and the discrete auxiliary equation (42)-(43), we have

$$
\begin{aligned}
& D_{h}\left(\left(E_{h}-\pi_{h} E-h^{2} \tilde{E}_{h}, H_{h}-I_{h} H-h^{2} \tilde{h}\right) ;(\phi, \psi)\right) \\
& =D_{h}\left(\left(E_{h}-E, H_{h}-H\right) ;(\phi, \psi)\right)+D_{h}\left(\left(E-\pi_{h} E, H-I_{h} H\right) ;(\phi, \psi)\right) \\
& -h^{2} D_{h}\left(\left(\tilde{E}_{h}, \tilde{H}_{h}\right) ;(\phi, \psi)\right) \\
& =<H, \phi \times \mathbf{n}>_{h}-h^{2} D_{h}\left(\left(\tilde{E}_{h}, \tilde{H}_{h}\right) ;(\phi, \psi)\right)+D_{h}\left(\left(E-\pi_{h} E, H-I_{h} H\right) ;(\phi, \psi)\right) \\
& =O\left(h^{4}\right)\|H\|_{5}\|\phi\|_{2 h}+O\left(h^{4}\right)\left(\|E\|_{4}+\|H\|_{4}\right)\left(\|\phi\|_{0}+\|\psi\|_{1 h}\right) \\
& =O\left(h^{4}\right)\left(\|E\|_{4}+\|H\|_{5}\right)\left(\|\phi\|_{2 h}+\|\psi\|_{2 h}\right) .
\end{aligned}
$$

$\forall f \in\left(L^{2}(\Omega)\right)^{3}$, there exists $(u, v) \in\left[\mathbf{H}_{\mathbf{0}}(\mathbf{c u r l}, \Omega) \bigcap\left(H^{2}(\Omega)\right)^{3}\right] \times\left(H^{2}(\Omega)\right)^{3}$ such that

$$
\begin{aligned}
& (\sigma \phi, u)-(\psi, \operatorname{curl} u)=(\phi, f), \quad \forall \phi \in \mathbf{H}_{\mathbf{0}}(\operatorname{curl}, \Omega), \\
& (i \omega \mu \psi, v)+(\operatorname{curl} \phi, v)=0, \quad \forall \psi \in\left(L^{2}(\Omega)\right)^{3} .
\end{aligned}
$$

with the regularity property [19]: $\|u\|_{2}+\|v\|_{2} \leq C\|f\|_{0}$.

Suppose that $\left(u_{h}, v_{h}\right)$ is the mixed nonconforming finite element solution of $(u, v)$, respectively, then

$$
\begin{array}{ll}
\left(\sigma \phi, u_{h}\right)_{h}-\left(\psi, \operatorname{curl} u_{h}\right)_{h}=(\phi, f), & \forall \phi \in V_{h}, \\
\left(i \omega \mu \psi, v_{h}\right)_{h}+\left(\operatorname{curl} \phi, v_{h}\right)_{h}=0, & \forall \psi \in W_{h} .
\end{array}
$$

Thus, we have

$$
D_{h}\left((\phi, \psi) ;\left(u_{h}, v_{h}\right)\right)=(\phi, f), \quad \forall \phi \in V_{h}, \psi \in W_{h} .
$$

Take $\phi=E_{h}-\pi_{h} E-h^{2} \tilde{E}_{h}, \psi=H_{h}-I_{h} H-h^{2} \tilde{H}_{h}$, we get

$$
\begin{aligned}
& \left(E_{h}-\pi_{h} E-h^{2} \tilde{E}_{h}, f\right) \\
& =D_{h}\left(\left(E_{h}-\pi_{h} E-h^{2} \tilde{E}_{h}, H_{h}-I_{h} H-h^{2} \tilde{H}_{h}\right) ;\left(u_{h}, v_{h}\right)\right) \\
& =O\left(h^{4}\right)\left(\|E\|_{4}+\|H\|_{5}\right)\left(\left\|u_{h}\right\|_{2 h}+\left\|v_{h}\right\|_{2 h}\right) .
\end{aligned}
$$


Note that

$$
\begin{aligned}
& \left\|u_{h}\right\|_{2 h} \leq\left\|u_{h}-\pi_{h} u\right\|_{2 h}+\left\|\pi_{h} u-u\right\|_{2 h}+\|u\|_{2} \\
& \leq C h^{-1}\left\|u_{h}-\pi_{h} u\right\|_{1 h}+C\|u\|_{2} \leq C\|u\|_{2} \leq C\|f\|_{0} \\
& \left\|v_{h}\right\|_{2 h} \leq C\|f\|_{0} .
\end{aligned}
$$

Therefore, $\forall f \in\left(L^{2}(\Omega)\right)^{3}$, there exists

$$
\left(E_{h}-\pi_{h} E-h^{2} \tilde{E}_{h}, f\right)=O\left(h^{4}\right)\left(\|E\|_{4}+\|H\|_{5}\right)\|f\|_{0}
$$

Let $f=E_{h}-\pi_{h} E-h^{2} \tilde{E}_{h}$, we have

$$
\left\|E_{h}-\pi_{h} E-h^{2} \tilde{E}_{h}\right\|_{0}=O\left(h^{4}\right)\left(\|E\|_{4}+\|H\|_{5}\right) .
$$

Similarly, $\forall g \in\left(L^{2}(\Omega)\right)^{3}$, there exists $(w, p) \in\left[\mathbf{H}_{\mathbf{0}}(\mathbf{c u r l}, \Omega) \bigcap\left(H^{2}(\Omega)\right)^{3}\right] \times$ $\left(H^{2}(\Omega)\right)^{3}$ such that

$$
\begin{array}{ll}
(\sigma \phi, w)-(\psi, \operatorname{curl} w)=0, & \forall \phi \in \mathbf{H}_{\mathbf{0}}(\operatorname{curl}, \Omega), \\
(i \omega \mu \psi, p)+(\operatorname{curl} \phi, p)=(\psi, g), & \forall \psi \in\left(L^{2}(\Omega)\right)^{3} .
\end{array}
$$

with the regularity property [19]: $\|w\|_{2}+\|p\|_{2} \leq C\|g\|_{0}$. Using the same technique, we have

$$
\left\|H_{h}-I_{h} H-h^{2} \tilde{H}_{h}\right\|_{0}=O\left(h^{4}\right)\left(\|E\|_{4}+\|H\|_{5}\right) .
$$

Now we can finish the proof.

Let $\tau=\bigcup_{i=1}^{64} K_{i} \in \mathcal{J}_{4 h}$ with $K_{i} \in \mathcal{J}_{h}$. The interpolation operator $\Pi_{4 h}^{3}$ and $I_{4 h}^{3}$ can be defined by:

$$
\begin{aligned}
& \Pi_{4 h}^{3} E \in Q_{333}(\tau), \quad I_{4 h}^{3} H \in Q_{333}(\tau), \\
& \int_{K_{i}}\left(\Pi_{4 h}^{3} E-E\right) d x d y d z=0, i=1,2, \cdots, 64 . \\
& \int_{K_{i}}\left(I_{4 h}^{3} H-H\right) d x d y d z=0, i=1,2, \cdots, 64,
\end{aligned}
$$


with the properties

$$
\begin{aligned}
& \Pi_{4 h}^{3} \pi_{h} E=\Pi_{4 h}^{3} E, \\
& \left\|\Pi_{4 h}^{3} v\right\|_{0} \leq\|v\|_{0}, \quad \forall v \in V_{h}, \\
& \left\|\Pi_{4 h}^{3} E-E\right\|_{0} \leq c h^{4}\|E\|_{4}, \forall E \in\left(H^{4}(\Omega)\right)^{3}, \\
& I_{4 h}^{3} I_{h} H=I_{4 h}^{3} H, \\
& \left\|I_{4 h}^{3} w\right\|_{0} \leq\|w\|_{0}, \quad \forall w \in W_{h}, \\
& \left\|I_{4 h}^{3} H-H\right\|_{0} \leq c h^{4}\|H\|_{4}, \forall H \in\left(H^{4}(\Omega)\right)^{3} .
\end{aligned}
$$

Theorem 5.2. Under the condition of Theorem 5.1, we have the following estimations,

$$
\begin{aligned}
& \left\|\Pi_{4 h}^{3} E_{h}-E-h^{2} \tilde{E}\right\|_{0}=O\left(h^{4}\right)\left(\|E\|_{4}+\|H\|_{5}\right), \\
& \left\|I_{4 h}^{3} H_{h}-H-h^{2} \tilde{H}\right\|_{0}=O\left(h^{4}\right)\left(\|E\|_{4}+\|H\|_{5}\right) .
\end{aligned}
$$

Proof: By Theorem 5.1, and the properties of interpolation operator $\Pi_{4 h}^{3}$, we have

$$
\begin{aligned}
& \Pi_{4 h}^{3} E_{h}-E-h^{2} \tilde{E}=\Pi_{4 h}^{3}\left(E_{h}-\pi_{h} E-h^{2} \tilde{E}_{h}\right) \\
& +\left(\Pi_{4 h}^{3} \pi_{h} E-E\right)+h^{2}\left(\Pi_{4 h}^{3} \tilde{E}_{h}-\tilde{E}\right) \\
& =\Pi_{4 h}^{3}\left(E_{h}-\pi_{h} E-h^{2} \tilde{E}_{h}\right)+\left(\Pi_{4 h}^{3} E-E\right) \\
& +h^{2}\left(\Pi_{4 h}^{3} \tilde{E}_{h}-\Pi_{4 h}^{3} \pi_{h} \tilde{E}\right)+h^{2}\left(\Pi_{4 h}^{3} \pi_{h} \tilde{E}-\tilde{E}\right) \\
& =O\left(h^{4}\right)\left(\|E\|_{4}+\|H\|_{5}\right)+h^{2} \Pi_{4 h}^{3}\left(\tilde{E}_{h}-\pi_{h} \tilde{E}\right)+h^{2}\left(\Pi_{4 h}^{3} \tilde{E}-\tilde{E}\right) \\
& =O\left(h^{4}\right)\left(\|E\|_{4}+\|H\|_{5}\right) .
\end{aligned}
$$

Here, we also need the superclose results and the regularity property of $\tilde{E}$. Therefore, we get (46). The same to (47). Then we can finish the proof.

In order to use extrapolation technique, we can divide each $K_{i} \in \mathcal{J}_{h}$ into 8 small congruent element $K_{i, j, k} \in \mathcal{J}_{h / 2},(i, j, k=1,2)$, and the corresponding nonconforming mixed finite element space is denoted by $V_{h / 2} \times W_{h / 2}$. Let $\left(E_{h / 2}, H_{h / 2}\right) \in V_{h / 2} \times W_{h / 2}$ and $\left(\Pi_{4 h}^{3}, I_{4 h}^{3}\right)$ be the finite element approximation and the interpolation operator with respect to the new partition.

With the help of Theorem 5.2, we can improve the accuracy by applying the Richardson extrapolation. 
Let $\check{E}_{h}=\Pi_{4 h}^{3} E_{h}, \check{H}_{h}=I_{4 h}^{3} H_{h}$. Compute $\left(E_{h}^{\text {extra }}, H_{h}^{\text {extra }}\right)$ by the following formulas:

$$
\begin{aligned}
E_{h}^{\text {extra }} & =\frac{4 \check{E}_{h / 2}-\check{E}_{h}}{3}, \\
H_{h}^{\text {extra }} & =\frac{4 \check{H}_{h / 2}-\check{H}_{h}}{3} .
\end{aligned}
$$

Theorem 5.3. Under the condition of Theorem 5.1, we have the following estimations to $\left(E_{h}^{\text {extra }}, H_{h}^{\text {extra }}\right)$,

$$
\begin{aligned}
& \left\|E_{h}^{\text {extra }}-E\right\|_{0}=O\left(h^{4}\right)\left(\|E\|_{4}+\|H\|_{5}\right), \\
& \left\|H_{h}^{\text {extra }}-H\right\|_{0}=O\left(h^{4}\right)\left(\|E\|_{4}+\|H\|_{5}\right) .
\end{aligned}
$$

Proof: First, we prove (50). By Theorem 5.2, we have

$$
\begin{aligned}
& 4 \check{E}_{h / 2}-\check{E}_{h}-3 E=4\left(\check{E}_{h / 2}-E-\left(\frac{h}{2}\right)^{2} \tilde{E}\right)-\left(\check{E}_{h}-E-h^{2} \tilde{E}\right) \\
& =O\left(h^{4}\right)\left(\|E\|_{4}+\|H\|_{5}\right) .
\end{aligned}
$$

Then (50) follows. The same to (51). Now we can finish the proof.

\section{Conclusion}

In this paper, we propose a new nonconforming mixed finite element approximation to the three-dimensional time-harmonic Maxwell's equations. By asymptotic expansion of interpolation error and nonconforming error, we can make a conclusion that this mixed finite element has superclose property. Global superconvergence property is also achieved by constructing a postprocess operator, which means it can improve the approximation order from $O(h)$ to $O\left(h^{2}\right)$ with boundary condition $E \times \mathbf{n}=0$. Furthermore, by constructing extrapolation operators, we can present $O\left(h^{4}\right)$ approximations in the sense of $L^{2}-$ norm.

\section{Acknowledgement}

The authors would like to express their grateful thanks to Professor Qun Lin at Chinese Academy of Sciences and Professor Tao Tang at Hong Kong Baptist University for their beneficial discussions. The first author is partially supported by Hong Kong Baptist university grant FRG/08-09/II-35. 


\section{References}

[1] H. Ammari and J.-C. Nédélec. Low-frequency electromagnetic scattering. SIAM J. Math. Anal., 31(4):836-861, 2000.

[2] Zhiqiang Cai, Jim Douglas, Jr., and Xiu Ye. A stable nonconforming quadrilateral finite element method for the stationary Stokes and NavierStokes equations. Calcolo, 36(4):215-232, 1999.

[3] Zhiqiang Cai and Xiu Ye. A mixed nonconforming finite element for linear elasticity. Numer. Methods Partial Differential Equations, 21(6):1043-1051, 2005.

[4] Zhiming Chen, Qiang Du, and Jun Zou. Finite element methods with matching and nonmatching meshes for Maxwell equations with discontinuous coefficients. SIAM J. Numer. Anal., 37(5):1542-1570 (electronic), 2000.

[5] P. Ciarlet, Jr. and Jun Zou. Fully discrete finite element approaches for time-dependent Maxwell's equations. Numer. Math., 82(2):193-219, 1999.

[6] Jim Douglas, Jr., Juan E. Santos, and Dongwoo Sheen. A nonconforming mixed finite element method for Maxwell's equations. Math. Models Methods Appl. Sci., 10(4):593-613, 2000.

[7] Graeme Fairweather, Qun Lin, Yanping Lin, Junping Wang, and Shuhua Zhang. Asymptotic expansions and Richardson extrapolation of approximate solutions for second order elliptic problems on rectangular domains by mixed finite element methods. SIAM J. Numer. Anal., 44(3):11221149, 2006.

[8] R. Hiptmair. Finite elements in computational electromagnetism. Acta Numer., 11:237-339, 2002.

[9] Andreas Kirsch and Peter Monk. A finite element/spectral method for approximating the time-harmonic Maxwell system in $\mathbf{R}^{3}$. SIAM J. Appl. Math., 55(5):1324-1344, 1995.

[10] Jichun Li. Error analysis of finite element methods for 3-D Maxwell's equations in dispersive media. J. Comput. Appl. Math., 188(1):107-120, 2006. 
[11] Jia-fu Lin and Qun Lin. Global superconvergence of the mixed finite element methods for 2-D Maxwell equations. J. Comput. Math., 21(5):637646, 2003.

[12] Qun Lin and Jichun Li. Superconvergence analysis for Maxwell's equations in dispersive media. Math. Comp., 77(262):757-771, 2008.

[13] Qun Lin and Jiafu Lin. Finite Element Methods:Accuracy and Improvement, Science Press, Beijing, 2006.

[14] Qun Lin, Lutz Tobiska, and Aihui Zhou. Superconvergence and extrapolation of non-conforming low order finite elements applied to the Poisson equation. IMA J. Numer. Anal., 25(1):160-181, 2005.

[15] Qun Lin and Ningning Yan. Construction and Analysis for Efficient Finite Element Methods (in Chinese).HeiBei University Press, 1996.

[16] Qun Lin and Ningning Yan. Global superconvergence for Maxwell's equations. Math. Comp., 69(229):159-176, 2000.

[17] Qun Lin, Changhui Yao, and Shanghui Jia. Asymptotic expansion and superconvergence analysis for maxwell's equations by nonconforming finte element methods. Submitted to J. Comput. Math.,2009.

[18] Ch. G. Makridakis and P. Monk. Time-discrete finite element schemes for Maxwell's equations. RAIRO Modél. Math. Anal. Numér., 29(2):171197, 1995.

[19] Peter Monk. A finite element method for approximating the timeharmonic Maxwell equations. Numer. Math., 63(2):243-261, 1992.

[20] Peter Monk. An analysis of Nédélec's method for the spatial discretization of Maxwell's equations. J. Comput. Appl. Math., 47(1):101-121, 1993.

[21] Peter Monk. Superconvergence of finite element approximations to Maxwell's equations. Numer. Methods Partial Differential Equations, 10(6):793-812, 1994.

[22] Peter Monk. A posteriori error indicators for Maxwell's equations. J. Comput. Appl. Math., 100(2):173-190, 1998. 
[23] Peter Monk. Finite element methods for Maxwell's equations. Numerical Mathematics and Scientific Computation. Oxford University Press, New York, 2003.

[24] J.-C. Nédélec. Mixed finite elements in $\mathbf{R}^{3}$. Numer. Math., 35(3):315$341,1980$.

[25] J.-C. Nédélec. A new family of mixed finite elements in $\mathbf{R}^{3}$. Numer. Math., 50(1):57-81, 1986.

[26] Juan E. Santos and Dongwoo Sheen. On the existence and uniqueness of solutions to Maxwell's equations in bounded domains with application to magnetotellurics. Math. Models Methods Appl. Sci., 10(4):615-628, 2000 .

[27] Dongyang Shi and Lifang Pei. Low order Crouzeix-Raviart type nonconforming finite element methods for approximating Maxwell's equations. Int. J. Numer. Anal. Model., 5(3):373-385, 2008.

[28] Martin Stynes and Lutz Tobiska. The streamline-diffusion method for nonconforming $Q_{1}^{\text {rot }}$ elements on rectangular tensor-product meshes. IMA J. Numer. Anal., 21(1):123-142, 2001.

[29] Hehu Xie. Extrapolation of the Nédélec element for the Maxwell equations by the mixed finite element method. Adv. Comput. Math., 29(2):135-145, 2008.

[30] Xiu Ye. Superconvergence of nonconforming finite element method for the Stokes equations. Numer. Methods Partial Differential Equations, 18(2):143-154, 2002. 